\title{
Commentary: Early to extubate, early to discharge-providing further evidence in favor of fast tracking and early recovery after surgery pathways
}

\author{
Bryan A. Whitson, MD, PhD, FACS
}

\footnotetext{
From the Division of Cardiac Surgery, Department of Surgery, Ohio State University, Columbus, Ohio. Disclosures: Author has nothing to disclose with regard to commercial support.

Received for publication March 29, 2019; accepted for publication March 29, 2019; available ahead of print May $31,2019$.

Address for reprints: Bryan A. Whitson, MD, PhD, FACS, COPPER Laboratory, Section of Thoracic Transplantation and Mechanical Circulatory Support, Division of Cardiac Surgery, Department of Surgery, The Ohio State University, N-816 Doan Hall, 410 W. 10th Ave, Columbus, OH 43210 (E-mail: bryan.whitson@ osumc.edu).

J Thorac Cardiovasc Surg 2020;159:191

$0022-5223 / \$ 36.00$

Copyright (C) 2019 by The American Association for Thoracic Surgery

https://doi.org/10.1016/j.jtcvs.2019.03.117
}

In this month's issue of the Journal, Dr Muller Moran and colleagues $^{1}$ from the University of Manitoba present data on the significant impact of relatively small differences in times to extubation on postoperative delirium and outcomes after isolated coronary artery bypass grafting. The authors took a slightly different approach than previous investigations and focused on the less than 24-hour time to extubation after cardiac surgery stratifying patients into less than 12-hour and a 12- to 24-hour cohorts. This focus on the early postoperative period provides us some significant insights into the benefits of fast extubation when clinically appropriate.

The primary end point relayed in the study was a significant positive impact on postoperative delirium in the shorter time to extubation cohort. In the multivariate model, there were additional confounders, although 12 to 24 hours of extubation was an independent predictor of postoperative delirium after coronary artery bypass grafting. Although the primary end point is significant in itself, there are other important takeaways from the study. First, there was an overall significantly shorter intensive care unit and hospital length of stay. Additionally, the need for reintubation and rates of postoperative pneumonia were significantly less. Furthermore, the proportion of patients being discharged to home was substantially larger in the early extubation cohort.

Although there are limitations of the study, this is a singleinstitution study, the analysis is retrospective, and the cohorts were somewhat different preoperatively; the overall impact of seemingly small differences in extubation times can be quite positive. The current study by Muller Moran and colleagues ${ }^{1}$ adds Canadian data to the initial US experience published in the Journal by Williams and colleagues, ${ }^{2}$ which demonstrated earlier recovery and improved quality. The overall Early Recovery After Surgery initiatives and Early Recovery After Surgery Cardiac Society ${ }^{3}$ work has challenged us, in a positive way, to take a deeper look at our

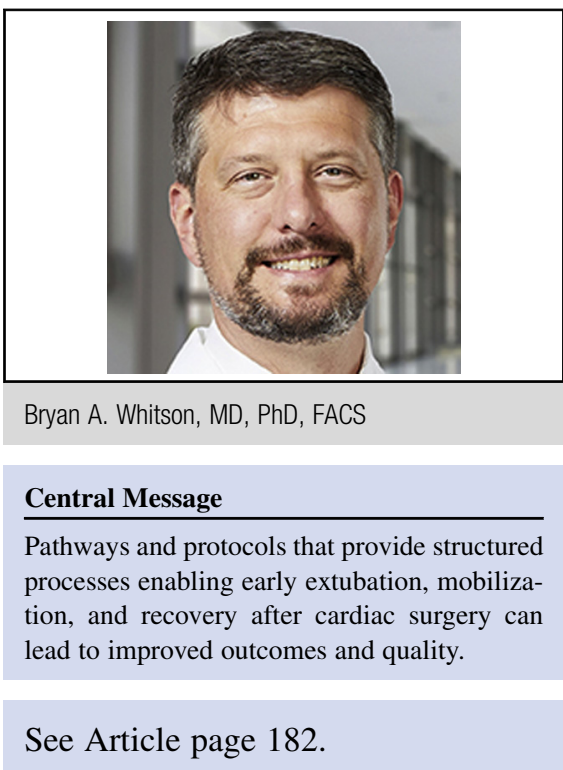

delivery of care models and strive to decrease variability and minimize deleterious effects of our care on the body's homeostasis. Increased mobility and earlier extubation are paramount. To continue to expand the positive effects of these initiatives, we need to continue to partner with our anesthesia colleagues $^{4}$ and the entire healthcare delivery team.

So that we are able to provide the best outcomes and highest quality cardiothoracic surgical care to our patients, we need to embrace the development of multimodal, multidisciplinary pathways and protocols that provide the structure for early extubation and early mobilization. The additional support of the current study by Muller Moran and colleagues ${ }^{1}$ may be the nudge for expanding multiinstitutional and multinational collaborations.

\section{References}

1. Muller Moran HR, Maguire D, Maguire D, Kowalski S, Jacobsohn E, Mackezie S et al. Association of earlier extubation and postoperative delirium after coronary artery bypass grafting. J Thorac Cardiovasc Surg. 2020;159:182-90.e7.

2. Williams JB, McConnell G, Allender JE, Woltz P, Kane K, Smith PK, et al. Oneyear results from the first US based enhanced recovery after cardiac surgery (ERAS Cardiac) program. J Thorac Cardiovasc Surg. December 8, 2018 [Epub ahead of print].

3. Early Recovery After Surgery Cardiac Society Expert Recommendations. Available at: https://www.erascardiac.org/recommendations/expert-recommendations. Accessed March 29, 2019

4. Noss C, Prusinkiewicz C, Nelson G, Patel PA, Augoustides JG, Gregory AJ. Enhanced recovery for cardiac surgery. J Cardiothorac Vasc Anesth. 2018;32 2760-70. 